\title{
Diseño y desarrollo de un sistema de alerta temprana para prevenir los efectos de flujos de detritos en la Quebrada Pedregal, Chosica - Lima
}

\section{Design and development of an early warning system to prevent the effects of debris flow in la Quebrada Pedregal, Chosica - Lima}

\author{
Giusepi Joel Gutiérrez Lavini', Víctor Manuel Uribe², Enrique Guadalupe Gómez ${ }^{3}$
}

Recibido: 02/08/2021 - Aprobado: 23/09/2021 - Publicado: 23/12/2021

\begin{abstract}
RESUMEN
Chosica es una zona con características especiales que hacen que se produzca flujos de detritos, la geología está compuesta por la superunidad Santa Rosa del Cretácico Superior, compuesta de afloramientos Tonalíticos y Granodioríticos, los cuales están cubiertos por depósitos aluviales pertenecientes al Cuaternario de la serie del Pleistoceno y Holoceno, las principales geoformas son quebrada y terrazas aluvionales compuesta por diversos flujos de detritos. En el presente trabajo se diseñó y desarrolló un sistema de alerta temprana por medio de una aplicación móvil (App) para prevenir a los pobladores de la quebrada Pedregal de los efectos generados por los huaicos mediante un sistema de alerta temprana en base a sensores pluviométricos, que miden la cantidad de precipitaciones caídas en un lugar durante un tiempo determinado y acelerómetros que pueda medir el grado de aceleración o movimiento. El aplicativo consta de una serie de interfaces, siendo las tres principales el de Bienvenida, el segundo el Mapa donde se pueden observar la ubicación del usuario y el de los sensores y el interfaz Cultura de Prevención, donde el usuario puede encontrar alternativas educativas sobre el huaico, cómo actuar ante ella y el sistema de la alerta. Este aplicativo se podría usar en otros tipos de fenómenos de remoción en masa.
\end{abstract}

Palabras claves: Flujo de detritos; sistema de alerta temprana; aplicación móvil; movimiento en masa; maqueta.

\begin{abstract}
Chosica is an area with special characteristics that cause debris flows, the geology is composed of the Santa Rosa superunit of the Upper Cretaceous, composed of Tonalitic and Granodioritic outcrops, which are covered by alluvial deposits belonging to the Quaternary of the Pleistocene series and Holocene, the main geoforms are streams and alluvial terraces composed of various debris flows. In the present work, an early warning system was designed and developed through a mobile application (App) to prevent the residents of the Pedregal stream from the effects generated by the huaicos through an early warning system based on rainfall sensors. They measure the amount of precipitation that has fallen in a place during a given time and accelerometers that can measure the degree of acceleration or movement. The application consists of a series of interfaces, the main three being the Welcome, the second the Map where you can see the location of the user and the sensors and the Prevention Culture interface, where the user can find educational alternatives on the huaico, how to act before it and the alert system. This application could be used in other types of mass movement phenomena.
\end{abstract}

Keywords: Debris flow; early warning system; mobile application; mass movement; mockup.

\footnotetext{
1 Universidad Nacional Mayor de San Marcos, Facultad de Ingeniería Geológica, Minera, Metalúrgica y Geográfica, Lima, Perú. Egresado. E-mail: ggutierrezlavini@gmail.com - ORCID: https://orcid.org/0000-0002-4938-344X

2 Universidad Nacional Mayor de San Marcos, Facultad de Ingeniería Geológica, Minera, Metalúrgica y Geográfica, Lima, Perú. Docente. E-mail: vuribec@unmsm.edu.pe - ORCID: https://orcid.org/0000-0002-3096-325X

3 Universidad Nacional Mayor de San Marcos, Facultad de Ingeniería Geológica, Minera, Metalúrgica y Geográfica, Lima, Perú. Docente. E-mail: eguadalupeg@unmsm.edu.pe - ORCID: https://orcid.org/0000-0001-9583-8807
} 


\section{INTRODUCCIÓN}

La ciudad de Chosica (Perú), se ubica al este de la provincia de Lima y la microcuenca de Pedregal es una de las quebradas de este distrito (Figura 1).

Sus habitantes, conviven con los flujos de detritos (huaicos), que es una problemática que data de hace muchos años y ha logrado constituirse como una amenaza permanente. Estos flujos de lodo y rocas se originan por fuertes precipitaciones pluviales en las quebradas en época de verano entre diciembre y marzo, convirtiéndose en flujos peligrosos generando cierre de vías, inundaciones, destrucción de viviendas, parálisis de actividades en la ciudad y el cobro de vidas humanas.

Las quebradas de Chosica son un claro ejemplo de una deficiente planificación urbana, estas fueron ocupadas por los moradores desde hace 41 años, al inicio de la década de los 80 , si a ello agregamos la inestabilidad del suelo y la pendiente de las laderas hacen que estos flujos de detritos sean muy peligrosos, porque el material aluvial que posee una capacidad portante baja, en presencia de agua, la fricción interna cede o llega a su límite y el material empieza a movilizarse, poniendo en riesgo a los moradores que se encuentran asentados en estos lugares. El flujo anual promedio del distrito de Chosica, que tiene un área de captación alrededor del $70 \%$, y de $32 \mathrm{~m}^{3} / \mathrm{s}$, concorde con el registro hídrico del lapso de tiempo desde inicios del año 1969 al 2019 (SENAMHI, 2018).

Los flujos de detritos incorporan una cantidad considerable de material saturado en su trayectoria al descender por el canal. Por último, son depositados en forma de abanicos (Chiroque \& Lavado, 2017).

Los flujos de detritos del año 1987, que fueron desplazados por la quebrada Pedregal, con un volumen cercano a los $650000 \mathrm{~m}^{3}$ de escombros, que fluyeron con una velocidad promedio aproximadamente de $11 \mathrm{~km} / \mathrm{h}$, en sus momentos de mayores descargas, movilizando enormes bloques empacadas de arena limosa.

En la actualidad las quebradas están ocupadas siendo las más importantes por estar más densamente pobladas la quebrada de Mariscal Castilla, Quirio, La Libertad, La Cantuta, El Pedregal, Santo Domingo, Corrales y Carossio. Siendo estas dos últimas de alto riesgo pues presentan pendientes muy pronunciadas en tramos cortos lo que genera alto riesgo para los pobladores asentados en su cercanía (Figura 2) (Canales \& Cornejo, 2014, p. 17).

La principal justificación de la investigación es de presentar un innovador sistema de alerta temprana y analizar el proceso del diseño y desarrollo de este, para alertar a la población de la quebrada Pedregal a través de una aplicación móvil instalada en sus celulares sobre los efectos generados por los huaycos que fluyen por dicha quebrada.

Como consecuencia de la problemática descrita, se han desarrollado diversos estudios y obras de ingeniería para mitigar el impacto de estos fenómenos naturales, así como el diseño y desarrollo de sistemas de alerta temprana muy básicos, los cuales no han propiciado un impacto significativo en la población. A esto se le suma el abandono

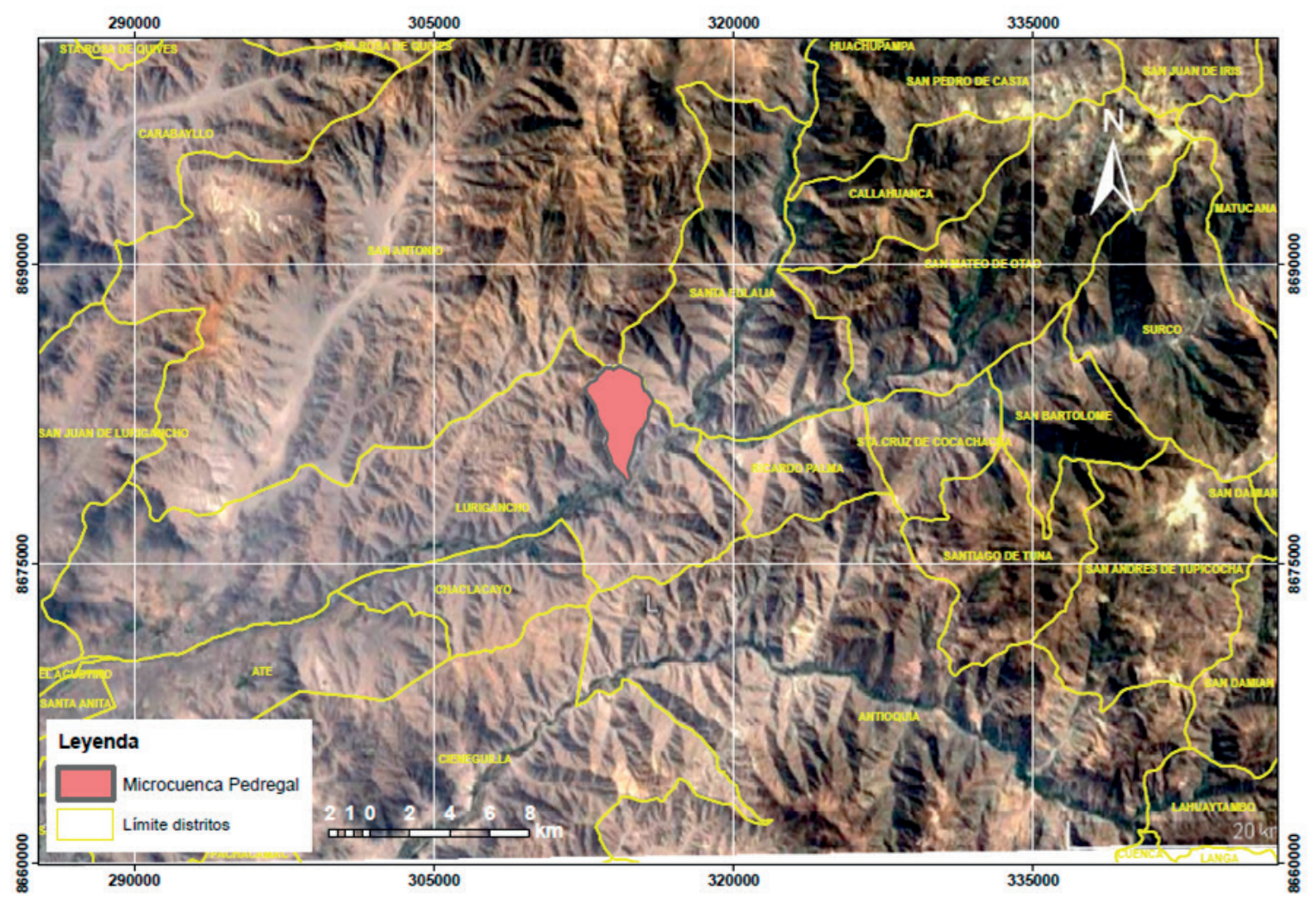

Figura 1. Ubicación de la microcuenca Pedregal. 


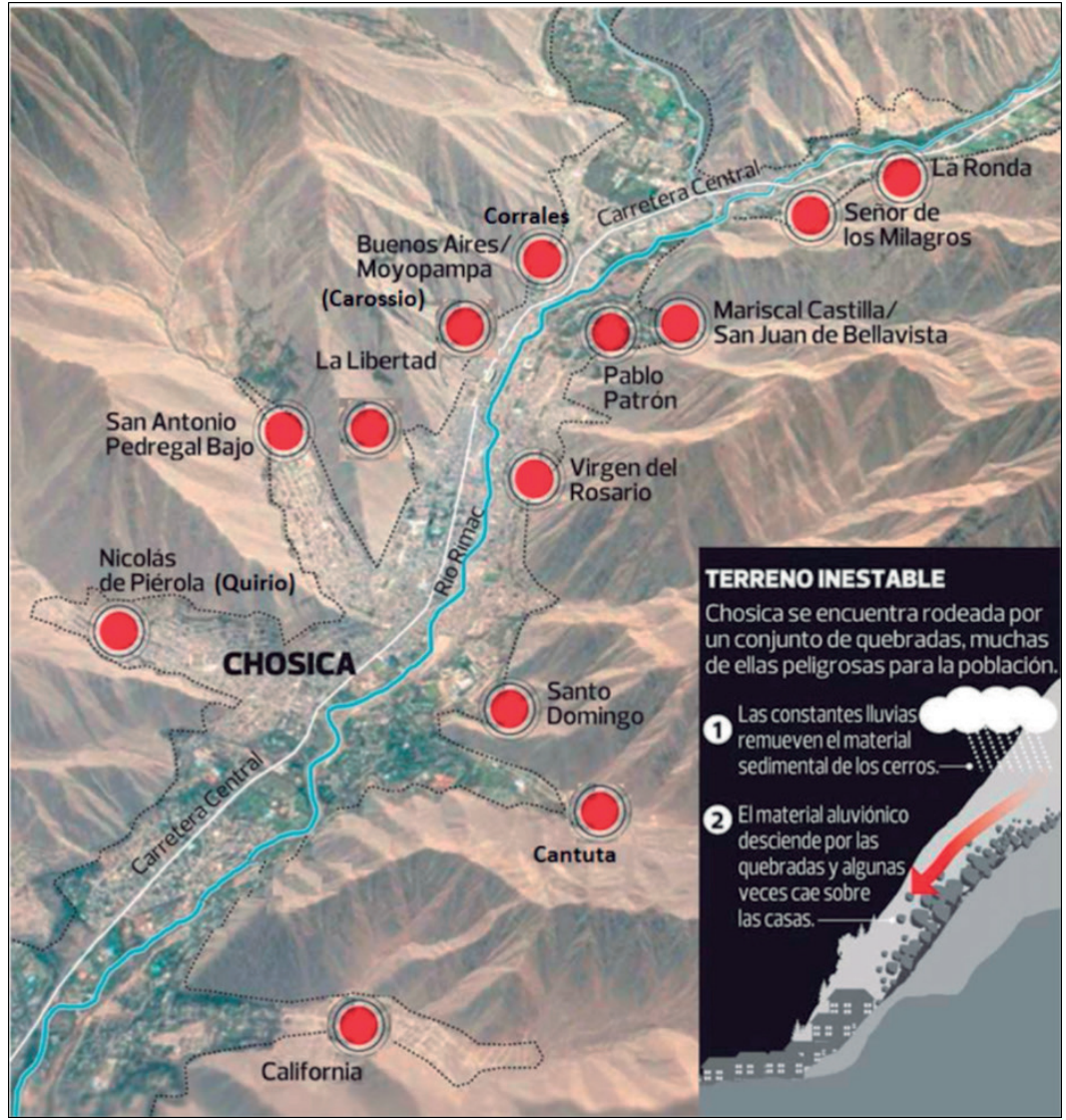

Figura 2. Quebradas y población afectadas por los Huaicos en Chosica. Tomado de Guadalupe G. \& Carrillo H. (2012, p.74).

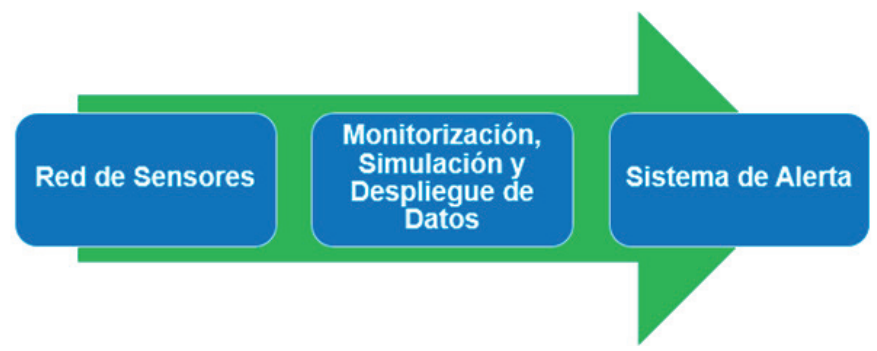

Figura 3. Diagrama de simulación. (Elaboración propia)

de las autoridades para tomar soluciones que conlleven a la reducción del riesgo.

Por tanto, este trabajo trata de dar una alternativa sencilla para reducir los daños humanos y/o materiales, presentando una solución mediante el uso de la tecnología, fomentando la cultura de la prevención y participación de los usuarios. La propuesta consiste en tener un mecanismo de alerta temprana con la que se pueda tomar decisiones en tiempo real para salvar vidas y prevenir daños colaterales. En relación con esto, se llevó a cabo la elaboración de una aplicación móvil para un S.A.T., que prevenga las consecuencias de los flujos de detritos.

Esta aplicación tiene como objetivo notificar a los usuarios que la instalen en sus dispositivos móviles, los niveles de peligrosidad de los diversos tramos de las quebradas presentes en la ciudad de Chosica, en tiempo real. Esta se diseñó bajo el sistema operativo Android, el más usado en Perú para dispositivos móviles. Asimismo, esta aplicación fue ejecutada en fases, dado que el propósito de esta aplicación es el desarrollo de sus funcionalidades. En ese sentido, se identificó, como modelo base, el desarrollo en cascada, puesto que esta permite al desarrollador enfocarse en mantener un orden metodológico de manera rigurosa para las etapas correspondientes, de tal manera, que el inicio de cada una debe esperar a la finalización del anterior, ajustándose el proceso de análisis, diseño y desarrollo del software de manera secuencial. En la (Figura 3), se muestra el diagrama de simulación del sistema de alerta temprana. 


\section{MATERIALES Y METODOS}

El presente trabajo se realizó principalmente en tres fases, una primera fase de gabinete, en la cual se da la recopilación de información bibliográfica, los planos topográficos, geología regional, análisis e interpretación de imágenes satelitales, revisión de estudios anteriores y un pre-inicio del diseño de la aplicación móvil. La segunda fase consistió en trabajo de campo reconocimiento la geología y geomorfología del área estudio. Se observó la situación actual de la quebrada, detallando las zonas más vulnerables, esta etapa se tuvo que realizar de manera muy rápida y eficiente, debido a la pandemia del COVID-19, con el ánimo de preservar la integridad del equipo. En esta fase, se adecuó el diseño de la aplicación para la zona de estudio, detallando lo que no se pudo realizar en la primera etapa y se priorizo realizar una descripción de formaciones litológicas superficiales con base en cortes naturales $\mathrm{y} / \mathrm{o}$ afloramientos superficiales, en donde se observan los depósitos de flujo de detritos ocurridos en la quebrada recientemente y determinar la posible ubicación de sensores remotos. En la tercera y última fase, se realizó una programación preliminar de la maqueta de la aplicación móvil y con los datos obtenidos en las etapas anteriores, que permitieron ajustar y corregir los posibles errores no detectados en las etapas previas, en la cual se analizó la información geológica y geotécnica del área, se evaluó las zonas críticas y se realizó la simulación del aplicativo. Finalmente, se realizaron las consultas necesarias a los asesores, fuentes bibliográficas y otros registros que ayudaron para el desarrollo de esta aplicación para este sistema de alerta temprana.

\section{RESULTADOS Y DISCUSIÓN}

Se pudo determinar que la microcuenca Pedregal posee un drenaje detrítico (Figura 4), observándose quebradas principales y secundarias, cuya importancia radica en la ubicación teórica de los sensores.

Además, con el uso de herramientas informáticas, se determinó que el perímetro de la subcuenca Pedregal es de $15.3 \mathrm{Km}$, y cubre un área de $10.4 \mathrm{~km}^{2}$ aproximadamente. Por otra parte, es importante destacar que la topografía permite ver las altitudes y geoformas las cuales sirvieron como mapa base para la investigación. (Figura 5)

Otro de los resultados sobresalientes de esta investigación es la caracterización geológica de la zona de estudio, la cuales han encontrado afloramientos de rocas Granodioritas y Tonalitas (Súper Unidad Sta. Rosa) que infrayacen a depósitos aluviales pertenecientes al Cuaternario Pleistoceno (Qp-al) y al Cuaternario reciente (Qr-al). Esto se evidencia en la columna litoestratigráfica levantada en la zona. (Figura 6).

De acuerdo con los registros históricos de las precipitaciones pluviales provista por la estación Chosica (SENAMHI, 2018), fue posible inferir que la subcuenca Pedregal presenta lluvias esporádicas a lo largo del periodo mayo-noviembre, aumentando las precipitaciones en el periodo diciembre-abril. Eso último, se estimó por medio de los resultados que se obtuvieron realizando la estadística del periodo 1989-2020 de la estación hidrológica automática denominada Chosica, que cuenta con registros

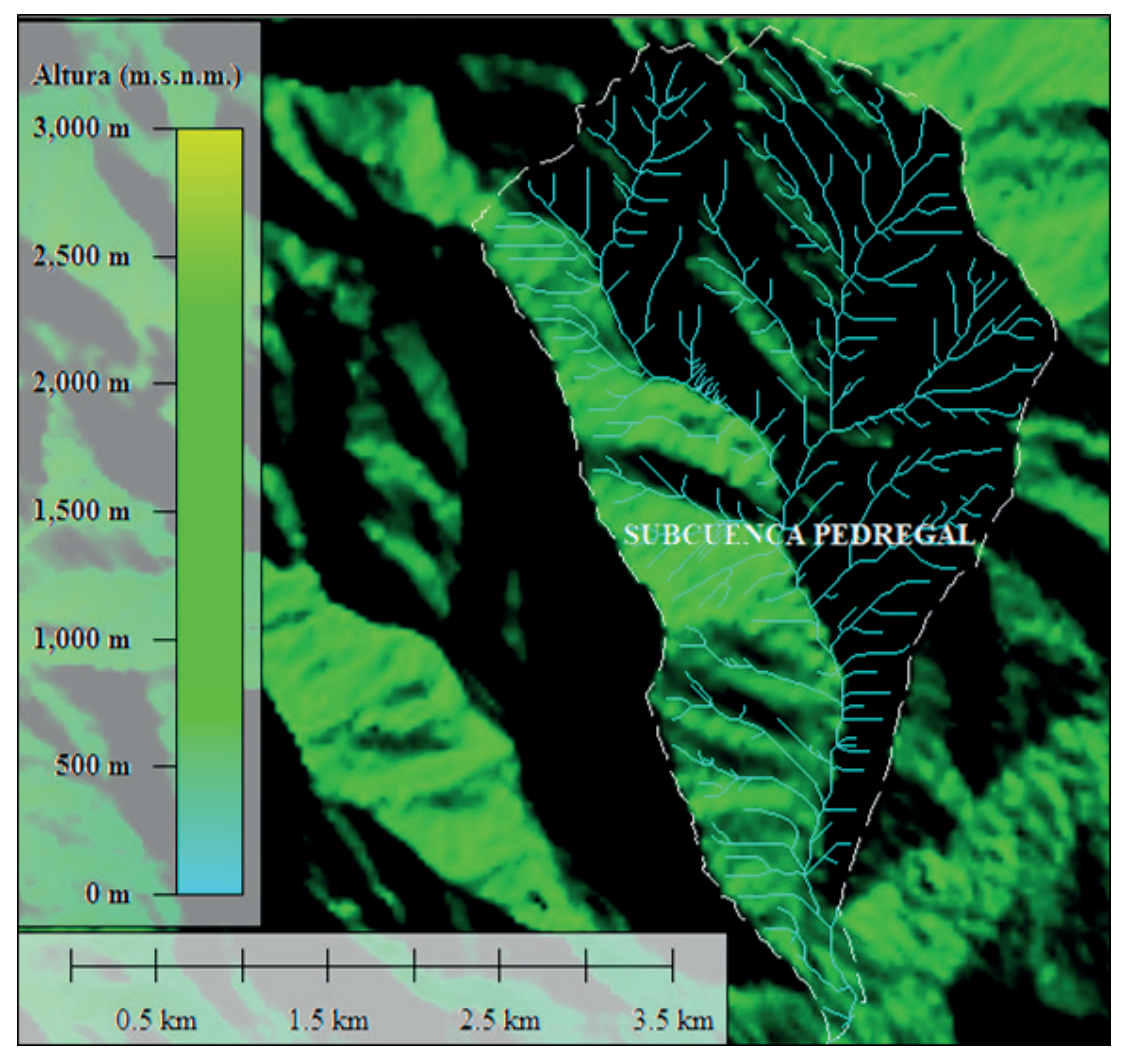

Figura 4. Drenaje detrítico de la microcuenca Pedregal. (Elaboración propia). 


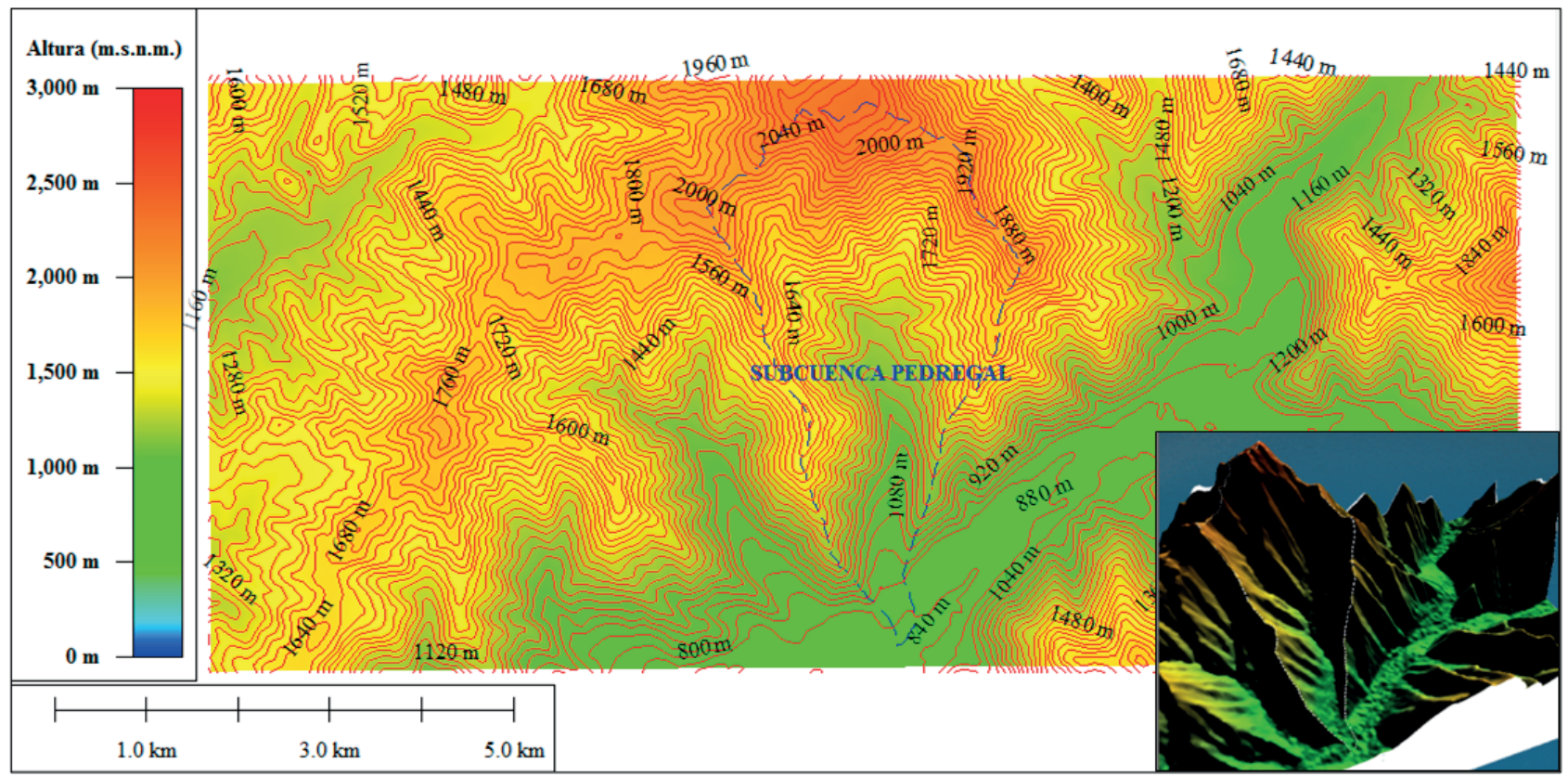

Figura 5. Topografía de la microcuenca Pedregal. (Elaboración propia)

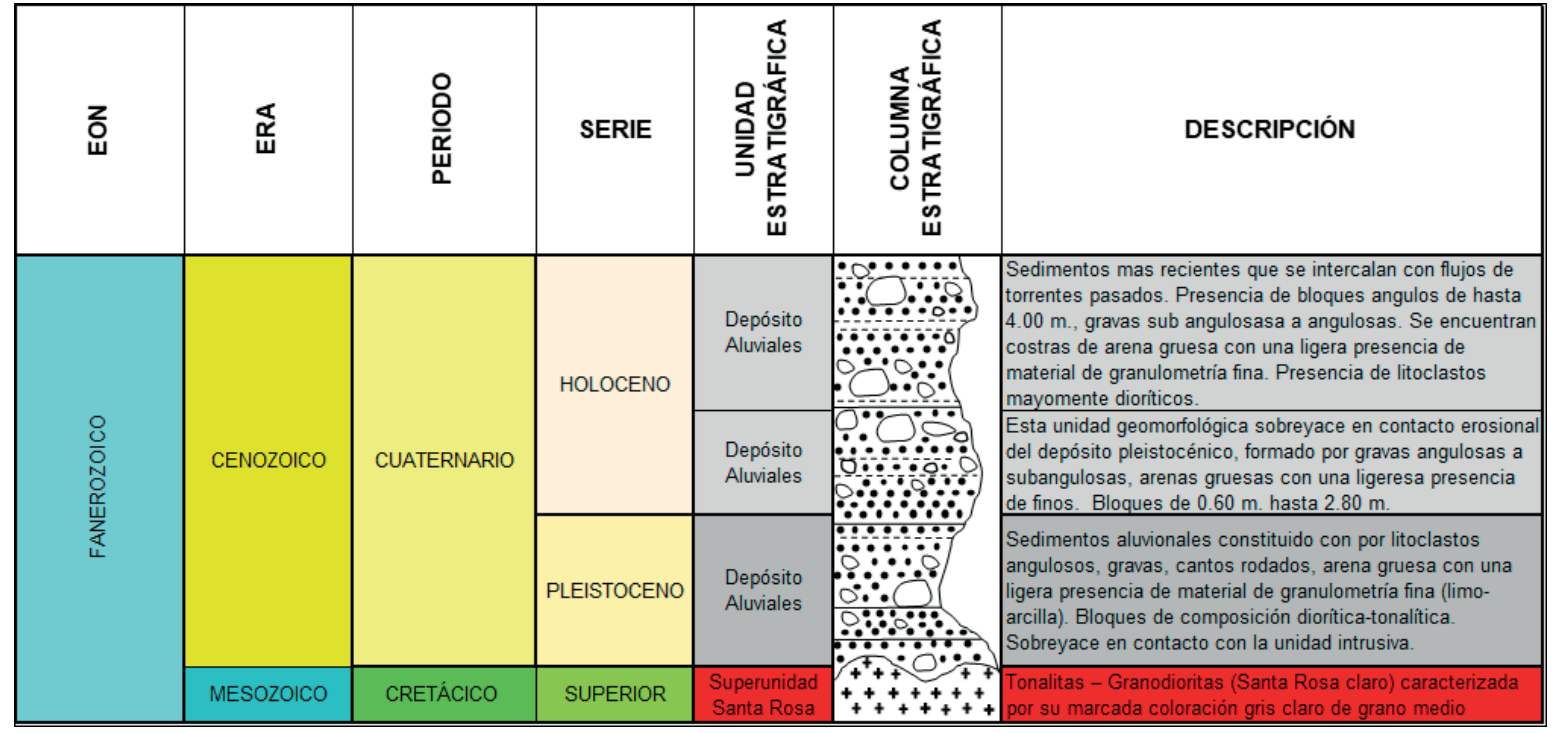

Figura 6. Columna litoestratigráfica de la geología local del área de estudio. (Elaboración propia)

de datos históricos desde hace más de 30 años. Asimismo, se determinó que las precipitaciones pluviales máximas ocurren en el mes de febrero con $4.30 \mathrm{~mm}$, y las mínimas en el mes de julio y septiembre con $0.1 \mathrm{~mm}$, respectivamente. En la Figura 7, se observa el registro de precipitaciones pluviales para el área de estudio.

De acuerdo con, la investigación realizada por Narciso Lira (2018) el determinó que para la subcuenca pedregal cuyo objetivo principal era el desarrollo de la gestión de inundación por caudales máximos en parte baja de la subcuenca, utilizando la información hidrometereológica desarrolló los límites pluviométricos y determinó las precipitaciones pluviales para los periodos de retorno de $5,10,15,20,50$ y 100 años y son $21.7,10,15,20,25,50$,
100 años y son $21.7,29.2,33.5,36.5,38.8,45.9,52.9 \mathrm{~mm}$ respectivamente, así como también los caudales máximos para los tiempos de retorno 5, 10, 15, 20, 25,50, 100 años y son $8,12,15,17,19,23,28 \mathrm{~m}^{3} / \mathrm{s}$. (p. 20 y 115). Esta información será de gran ayuda para el diseño del S.A.T. para determinar los algoritmos de desarrollo del tiempo de respuesta para la aplicación, sin embargo, teóricamente las notificaciones y alertas deben ser informados a los usuarios en tiempo real cuando los sensores pluviométricos y acelerómetros converjan en una sola respuesta la cual es, que un flujo de detritos se haya formado.

El análisis de la zona de estudio nos permitió usar un novedoso sistema de alerta temprana, en base a sensores pluviométricos, que miden la cantidad de precipitaciones 


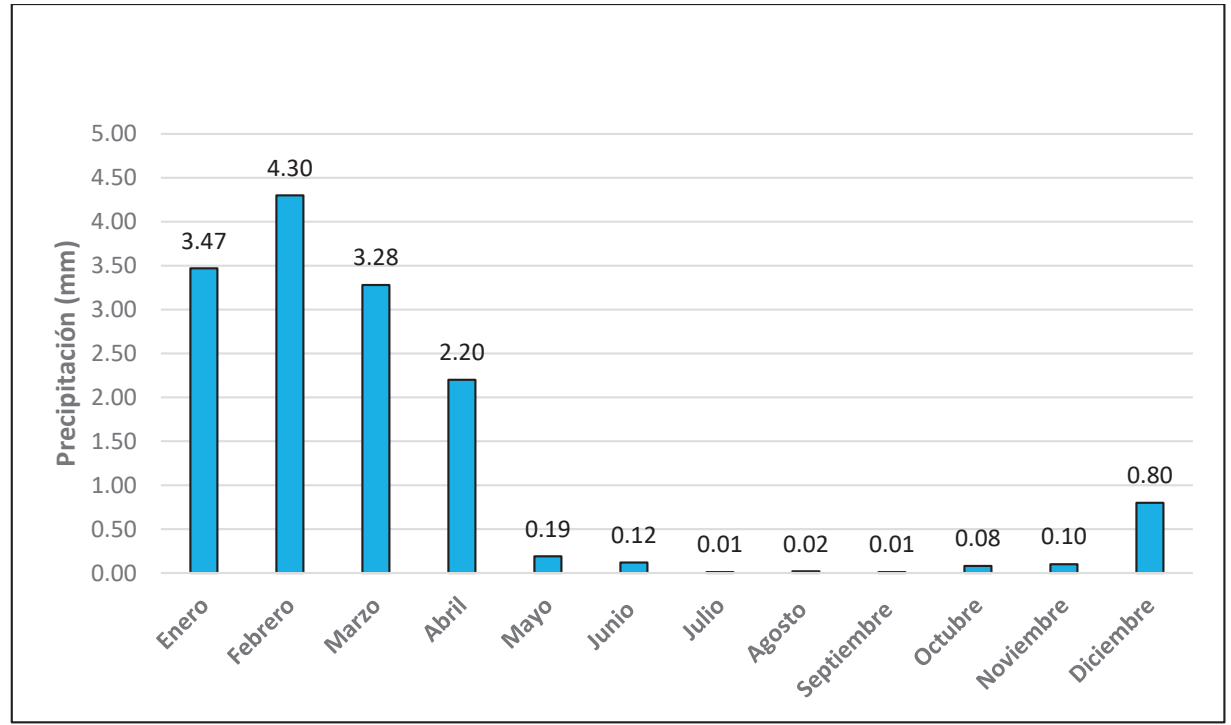

Figura 7. Registro de precipitaciones pluviales $(\mathrm{mm})$ promedio de la estación hidrológica Chosica (19892020). (Elaboración propia)

caídas en un lugar durante un tiempo determinado y acelerómetros que pueda medir el grado de aceleración o movimiento, buscando componentes que tengan la capacidad de medir y registrar los valores de aceleración y posición, y estos son celulares inteligentes o actualmente conocidos como smartphones, siendo lo novedoso que se pueden reutilizar sus componentes para el uso de estos sensores. Con el uso de una aplicación móvil se puede registrar los datos del acelerómetro desde un dispositivo móvil (Figura 8).

La ubicación de los sensores es fundamental para un sistema de alerta temprana, estos deben están distribuidos estratégicamente para que puedan recibir, analizar y comunicar la data registrada en un tiempo de respuesta determinado y de manera oportuna, para lo cual es necesario considerar los siguientes requisitos para una buena ubicación de un sensor:

- La ubicación del sensor debe ser colocado en un lugar donde sea óptimo el registro del parámetro a recopilar (monitoreo pluviométricos $\mathrm{y}$ acelerómetro). Asimismo, debe estar cercano a una fuente de energía y con un acceso cómodo al sistema de comunicación (internet).

- El sitio del sensor debe ser seguro para registrar el flujo de detritos evitando la pérdida y/o destrucción del instrumento. Además, debe estar protegido contra robos o daños ocasionados por terceros.

- Las redes inalámbricas de sensores deben ser capaces de albergar redes mucho más grandes y de comunicarse a intervalos regulares de datos. El reto consiste, por tanto, en proporcionar un bajo consumo, sin sacrificar la fiabilidad o la disponibilidad de la red.
- Las rutas hacia la ubicación de los sensores deben ser de fácil acceso, para garantizar el funcionamiento adecuado de los recursos tecnológicos. Adicionalmente, es imprescindible realizar un mantenimiento regular o realizar un cambio de un sensor en caso de ser necesario.

- La ubicación de los sensores debe formar secciones a lo largo de los 5.0 kilómetros aproximadamente de la quebrada Pedregal, formando una línea longitudinal a través de esta.

- Los sensores (acelerómetros) deben estar localizados en las siguientes coordenadas de la microcuenca Pedregal Tabla 1 (Figura 9)

Tabla 1. Ubicación teórica de los sensores a lo largo de la quebrada Pedregal

\begin{tabular}{|c|c|c|c|c|c|}
\hline \multicolumn{6}{|c|}{ Ubicación de sensores (Teórico) } \\
\hline \multirow{2}{*}{ N. ${ }^{\circ}$} & \multirow{2}{*}{ Nombre } & \multicolumn{4}{|c|}{ Coordenadas } \\
\hline & & Norte & Este & Cota (m.s.n.m.) & Datum \\
\hline 1 & Sensor A & 8679127 & 314736 & 839 & \multirow{6}{*}{ WGS 84} \\
\hline 2 & Sensor B & 8679284 & 314620 & 855 & \\
\hline 3 & Sensor C & 8679701 & 314496 & 901 & \\
\hline 4 & Sensor D & 8680364 & 314629 & 951 & \\
\hline 5 & Sensor $F$ & 8681064 & 314739 & 1043 & \\
\hline 6 & Sensor G & 8681536 & 314550 & 1092 & \\
\hline
\end{tabular}

Asimismo, la importancia de la visualización del sistema de alerta temprana por medio de una aplicación móvil (App) radica en la conexión que puede tener o no con el usuario, especialmente, con las personas que viven en la quebrada Pedregal. La relación debe ser amigable. Por consiguiente, la interfaz del usuario es una parte fundamental de las aplicaciones Android. El usuario puede comunicarse e interactuar con la App Huai.Co. Por lo cual, 


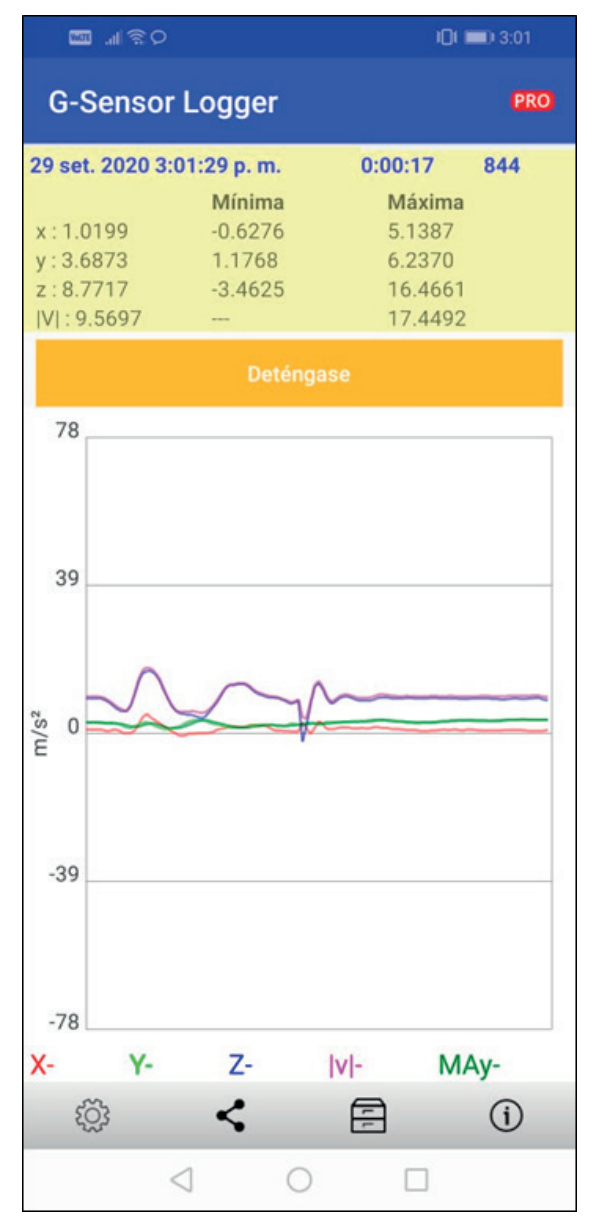

Figura 8. Aplicación móvil que registra los datos del acelerómetro del dispositivo celular. (Elaboración propia)

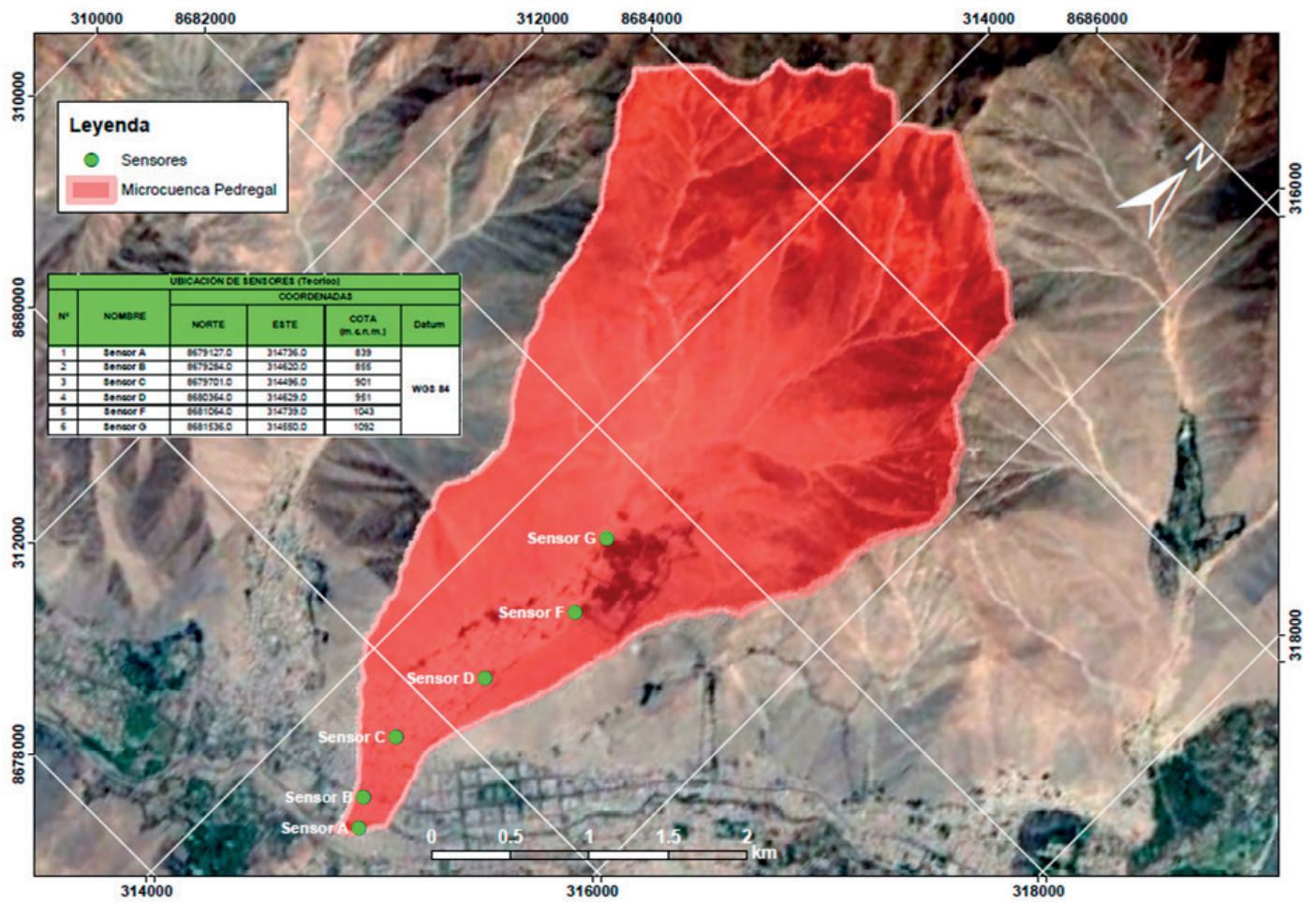

Figura 9. Ubicación teórica de los sensores (acelerómetros) en la microcuenca Pedregal. (Elaboración propia) 
se posee una serie de controles de selección que ofrece Android para mostrar e interactuar con la información en distintos formatos: listas, tablas, checks, botones, listas desplegables y galerías (Academia Android, 2015). A continuación, se presentan las diferentes interfaces más resaltantes de esta aplicación que el usuario podrá observar al momento de acceder a la aplicación móvil.

\subsection{Interfaz de bienvenida de la aplicación}

Esta es la página de bienvenida de la aplicación desarrollada Huai.Co. Esta va a ser la primera interacción del usuario con la aplicación, tendrá una duración de dos segundos. (Figura 10).

\subsection{Interfaz de la pestaña Mapa}

Aquí el usuario podrá visualizar su ubicación en tiempo real dentro de la subcuenca Pedregal, así como la ubicación teórica de los sensores y las respectivas coordenadas de cada uno de estos. (Figura 11).

\subsection{Interfaz de la pestaña Cultura de Prevención}

Esta pestaña es una de las más importantes en el diseño de esta aplicación, puesto que uno de los objetivos principales de esta investigación es fomentar la cultura de prevención en la población de la quebrada Pedregal, y a todo a aquel que teóricamente descarga esta aplicación. Esta pestaña es de carácter informativo, en la cual el usuario, de forma interactiva, podrá recibir consejos de cómo prepararse antes de un flujo de detritos, cómo actuar durante este fenómeno natural y cómo brindar su apoyo en los quehaceres de rehabilitación. En la opción de "Recomendaciones", podrá observar los números telefónicos de emergencia de la Policía Nacional, Bomberos, INDECI, entre otros; así como también podrá visualizar el mapa de rutas de evacuación

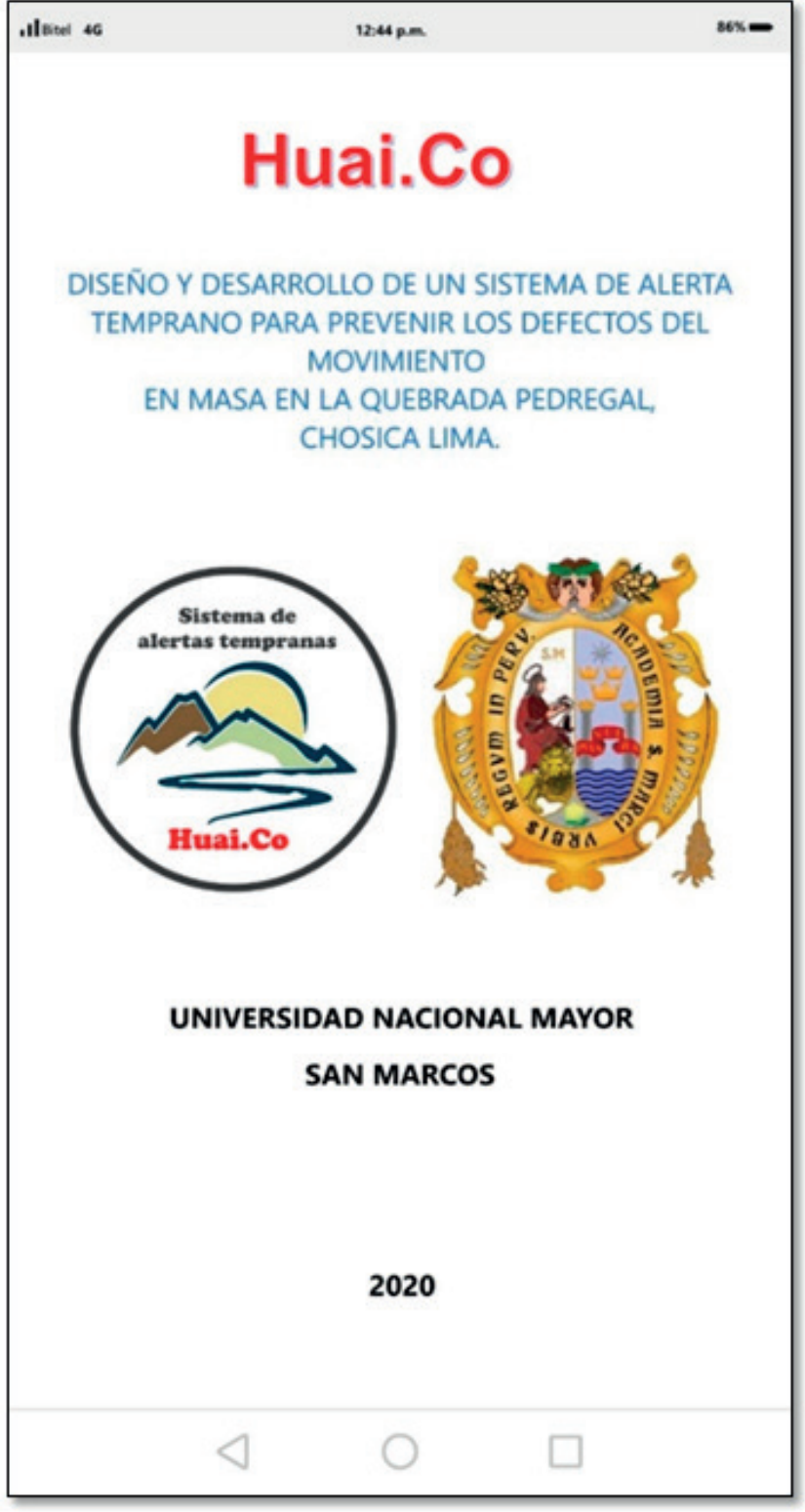

Figura 10. Pantalla de bienvenida a la aplicación Huai.Co. (Elaboración propia)

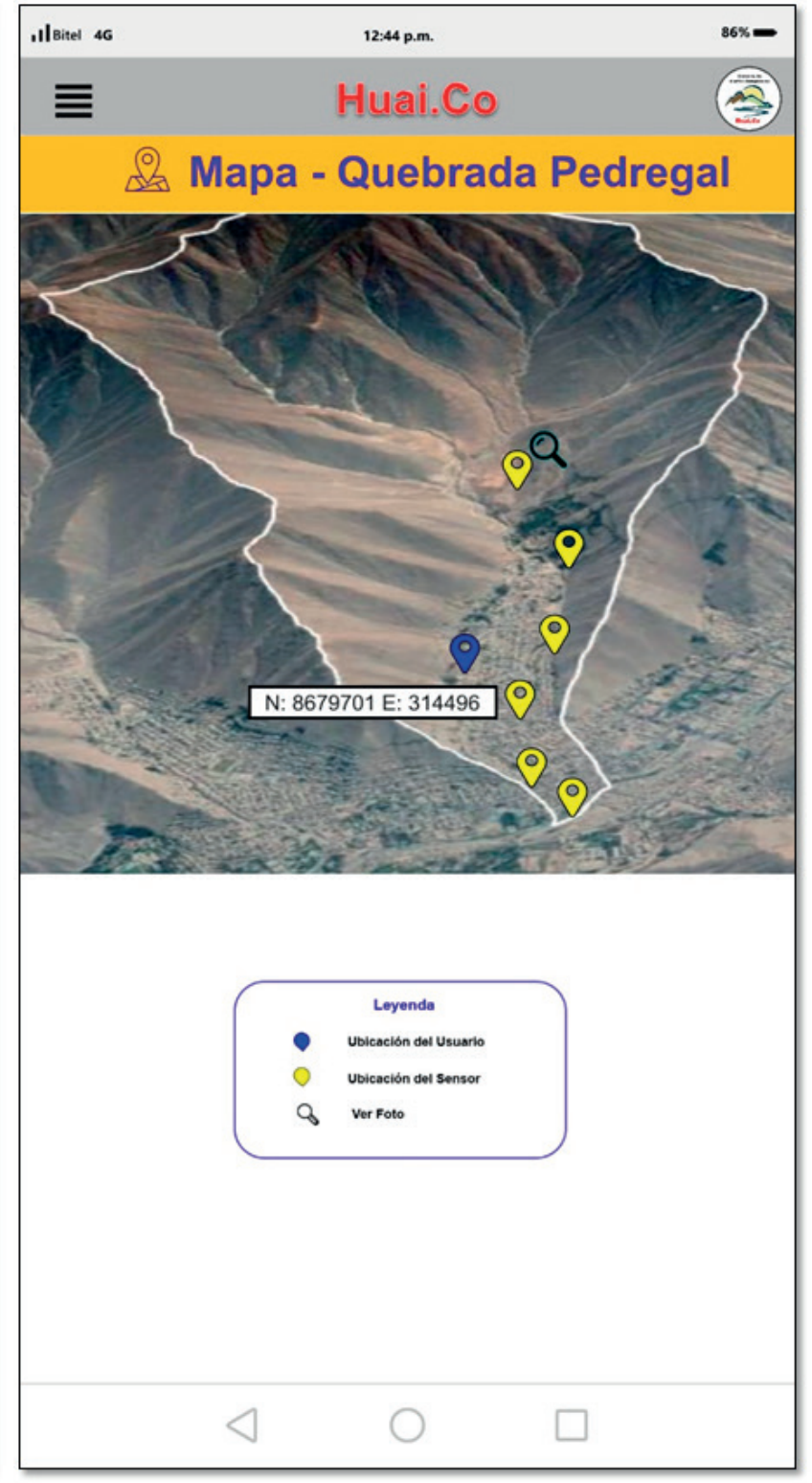

Figura 11. Pestaña de mapa, ubicación de usuario y sensores. (Elaboración propia) 
(ANA, 2016). Se presenta en pantalla la pestaña "Cultura de Prevención". (Figura 12)

De acuerdo con las estadísticas el uso de este medio de comunicación señala que hay unos 38 millones de líneas móviles que supera a la actual población peruana, asimismo, el 90.7\% de los dispositivos móviles en el Perú usan el sistema operativo Android (Yi Min Shum, 2020, p. 1), en base a esta información se optó elaborar este aplicativo bajo este sistema operativo.

De los resultados que fueron obtenidos durante el estudio, se puede concluir que se ha cumplido con el objetivo general de diseñar y desarrollar un S.A.T. para alertar los efectos de los flujos de detritos en base a los aspectos geológicos y geotécnicos en binomio con el uso de la tecnología de comunicación. Este sistema de alerta temprana es innovador, ya que considera el uso de las tecnologías actuales para la difusión de la información y alertas a los usuarios y pobladores de la quebrada Pedregal, que permite que la transmisión de estas no solo sea local, sino regionales y hasta nacionales.

El diseño de la aplicación móvil (mock up y/o maqueta) como parte fundamental de este sistema de alerta temprana, ha permitido establecer, que si es posible el uso de estos medios de comunicación para alertar una determinada población y de acuerdo con las estadísticas presentadas el uso del internet a través de las redes móviles permite una transmisión en tiempo real sobre el estado de la quebrada Pedregal.

Por ende, los resultados de esta investigación, establecen que el uso de este método de comunicación para este sistema de alerta temprana puede ser replicado para otras quebradas adyacentes a la quebrada Pedregal, asimismo, el uso de los sensores pluviométricos con sus respectivos umbrales determinados, pueden identificar un posible inicio de un flujo de detritos y a la vez nuestros acelerómetros instalados en nuestra quebrada principal de la microcuenca del Pedregal permitiría establecer el detonante para la activación de nuestro sistema de alerta temprana, el cual se encargaría en la transmisión de información y alerta a los pobladores de la quebrada Pedregal, con esto se podría mitigar y alertar sobre los efectos del movimiento en masa. En el siguiente link muestra la simulación de esta aplicación móvil Huai.Co: https://xd.adobe.com/view/ebc2c483-eea6-4163-88bf2 cf3c9c539e9-be91

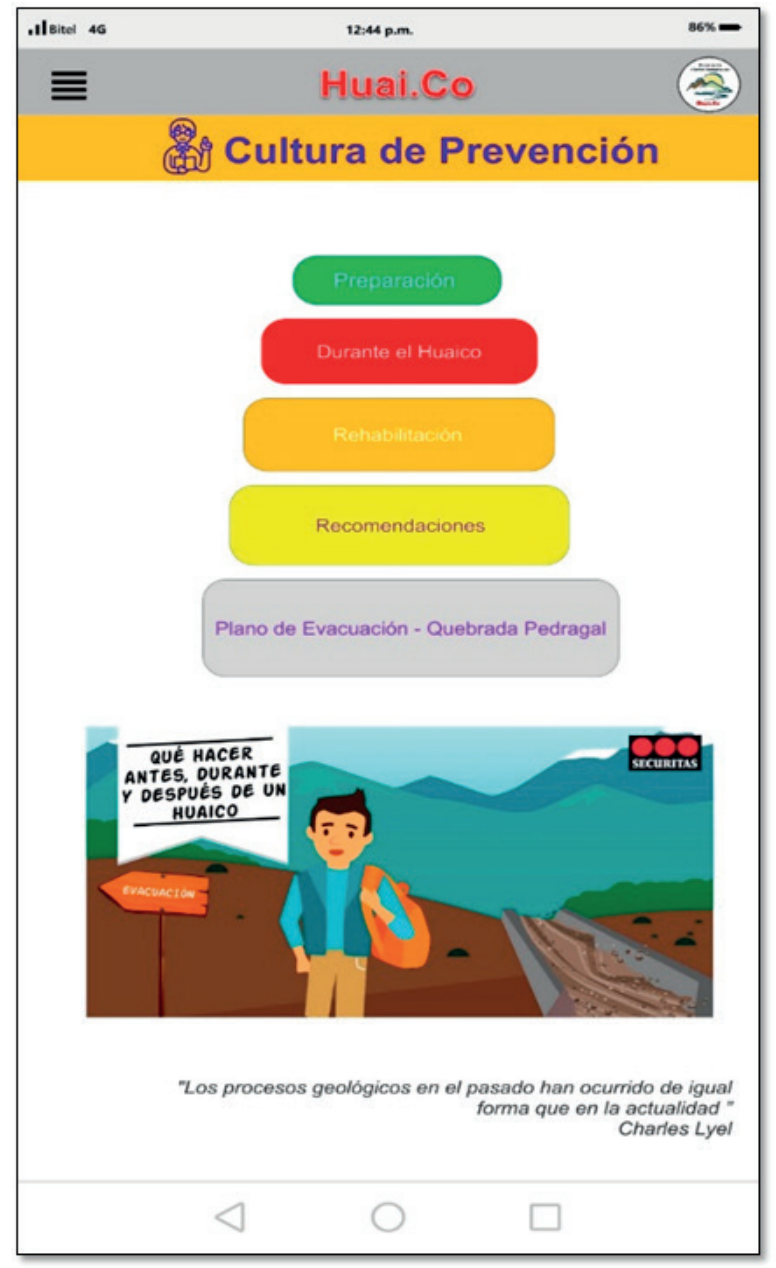

Figura 12. Pantalla de la pestaña "Cultura de Prevención". (Elaboración propia) 


\section{CONCLUSIONES}

Se logró diseñar y desarrollar un S.A.T., a través de una aplicación para dispositivos móviles, cuya función principal es alertar a los usuarios de la quebrada Pedregal y el estado de este en relación con los flujos de detritos "huaicos" en tiempo real. Para lo cual los sensores fueron localizados en puntos estratégicos de la quebrada Pedregal, este sistema está en base a sensores pluviométricos, que miden la cantidad de precipitaciones caídas en un lugar durante un tiempo determinado y acelerómetros que pueda medir el grado de aceleración o movimiento. El aplicativo consta de una serie de interfaces, siendo las tres principales el de Bienvenida, el segundo el Mapa donde se pueden observar la ubicación del usuario y el de los sensores y el interfaz de Cultura de Prevención donde el usuario puede encontrar alternativas educativas sobre el huaico.

La unidad geológica más resaltantes de la quebrada Pedregal, es la superunidad Santa Rosa del Cretácico Superior compuesta de afloramientos tonalíticos y granodioríticos, los cuales están cubiertos por depósitos aluviales pertenecientes al Cuaternario de la serie del Pleistoceno y Holoceno; asimismo, se realizó la caracterización de las unidades geomorfológicas, siendo la geoforma quebrada como la principal y las terrazas aluvionales, descritas en ambas márgenes de las quebradas depositadas por diversos flujos de detritos desde el Pleistoceno al Holoceno. Actualmente la quebrada Pedregal está en un proceso de ensanchamiento y profundización debido procesos de intemperismo, erosión y transporte de los flujos de detritos.

La innovación de las tecnologías actuales de los sistemas de comunicación, tienen una relación directa con las nuevas generaciones de usuarios, lo que permitirá siempre una mejora continua en los futuros sistemas de alerta temprana y por consiguiente, la innovación del uso de estos sistemas para otros tipos de desastres naturales.

\section{REFERENCIAS}

Academia Android. (2015). Elementos y diseño de Interfaz de Usuario en Apps Android. Digital Learnign LS. https:// academiaandroid.com/elementos-y-diseno-de-interfaz-deusuario-en-apps-android/

ANA. (2015). Mapa de Rutas de Evacuación. Autoridad Nacional Del Agua; Autoridad Nacional del Agua.

Canales, D., \& Cornejo, Y. (2014). Influencia de la Geotecnia en la Vulnerabilidad de las quebradas Secas en Chosica [Universidad Ricardo Palma]. https://repositorio.urp. edu.pe/bitstream/handle/URP/2175/canales_decornejo_ ys.pdf? sequence $=1 \&$ isAllowed $=\mathrm{y}$

Chiroque, C., \& Lavado, H. (2017). Aspectos geológicos y geodinámicos de flujos aluvionales en la quebrada Jicamarca y análisis de la vulnerabilidad para la implementación de un Sistema de Alerta Temprana (SAT) en el centro poblado Cajamarquilla. Revista Del Instituto de Investigación de La Facultad de Ingeniería Geológica, Minera, Metalurgica y Geográfica, 19(38), 47-55. https://doi.org/10.15381/iigeo. v19i38.13567

Guadalupe G., E., \& Carrillo H., N. (2012). Caracterización y análisis de los huaycos del 5 de abril del 2012 ChosicaLima. Revista Del Instituto de Investigación de La Facultad de Ingeniería Geológica, Minera, Metalurgica y Geográfica, 15(29), 69-82. https://revistasinvestigacion.unmsm.edu.pe/ index.php/iigeo/article/view/2206/1917

Narciso Lira, J. D. (2018). Gestión De Inundación Por Caudales Máximos En La Parte Baja De La Quebrada Pedregal Chosica [Universidad Nacional Federico Villarreal]. http:// repositorio.unfv.edu.pe/handle/UNFV/2540

SENAMHI. (2018). SENAMHI - Perú. Ministerio Del Ambiente. https://www.senamhi.gob.pe/servicios/?p=descarga-datosmeteorologicos

Yi Min Shum. (2020). Situación digital, Internet y redes sociales Perú 2020. Estudios Del Consumidor, Infografía. https:// yiminshum.com/redes-sociales-peru-2020/ 